\title{
Cholesteryl ester storage disease
}

INSERM

\section{Source}

INSERM. (1999). Orphanet: an online rare disease and orphan drug data base. Cholesteryl ester storage disease. ORPHA:75234

Cholesteryl ester storage disease (CESD) is a very rare, late-onset, genetic endocrine disease characterized by deficient or inactive lysosomal acid lipase (LAL) causing lipid build-up, which leads to atherosclerosis, hepatomegaly, splenomegaly, prog ressive liver disease, and malabsorption. 